Check for updates

Cite this: RSC Adv., 2018, 8, 10898

Received 6th February 2018

Accepted 9th March 2018

DOI: $10.1039 / c 8 \mathrm{ra01162a}$

rsc.li/rsc-advances

\title{
Cytoprotective effects of a tripeptide from Chinese Baijiu against AAPH-induced oxidative stress in HepG2 cells via Nrf2 signaling $\dagger$
}

\author{
Jihong Wu, ${ }^{\text {ab }}$ Baoguo Sun, ${ }^{a}$ Xuelian Luo, ${ }^{c}$ Mouming Zhao, (D) ${ }^{b}$ Fuping Zheng, ${ }^{a}$ \\ Jinyuan Sun, ${ }^{a}$ Hehe $\mathrm{Li}^{a}{ }^{a}$ Xiaotao Sun ${ }^{a}$ and Mingquan Huang (D) *a
}

\begin{abstract}
Antioxidant peptides have been widely reported, whereas the intracellular antioxidant activity of a tripeptide (Pro-His-Pro, PHP), which was newly isolated and identified from Chinese Baijiu in our previous study, are still poorly understood. This study investigated the protective effects of PHP on 2,2'-azobis (2-methylpropanimidamidine) dihydrochloride (AAPH)-induced oxidative stress in HepG2 cells and the involved molecular mechanisms. Pretreatment with PHP suppressed the generations of reactive oxygen species (ROS), malondialdehyde (MDA) and oxidized glutathione (GSSG), prevented a decrease in reduced glutathione $(\mathrm{GSH})$, and up-regulated the activities of cellular antioxidant enzymes. Moreover, PHP treatment stimulated the mRNA and protein expression levels of antioxidant enzymes and nuclear factor E2 related factor 2 (Nrf2). Meanwhile, PHP markedly reduced the level of Kelch-like ECH-associated protein 1 (Keap1), suggesting that PHP effectively activated Nrf2/antioxidant response element (ARE)mediated activity. These findings provide the first molecular basis for the health-promoting effects of $\mathrm{PHP}$ to prevent $\mathrm{AAPH}$-induced oxidative stress.
\end{abstract}

\section{Introduction}

Oxidative stress induced by the accumulation of reactive oxygen species (ROS) not only cause lipid oxidation in food systems but also cause human diseases. Nuclear factor E2 related factor 2 (Nrf2), a key regulator of antioxidant signaling, binds to Kelchlike ECH-associated protein 1 (Keap1) under homeostatic conditions within the cytoplasm. ${ }^{1}$ When oxidative or chemical stresses or Nrf2 activators appear, the Nrf2/Keap1 complex is dissociated and then Nrf2 translocate into the nucleus, where it promotes the transcription activation of phase II detoxifying/ antioxidant enzymes by binding to antioxidant response element (ARE) in the promoter regions of the target genes., ${ }^{2,3}$ Compounds that undergo redox cycling to generate ROS can affect the Nrf2-ARE transcriptional pathway., ${ }^{4,5}$ Therefore, activation of the Nrf2-ARE signaling pathway is a major mechanism currently in the cellular to defense against oxidative stress by

${ }^{a}$ Beijing Advanced Innovation Center for Food Nutrition and Human Health, Beijing Technology and Business University, Beijing 100048, China. E-mail: hmqsir@163. com; Tel: $+86-10-68985382$

${ }^{b}$ School of Food Science and Engineering, South China University of Technology, Guangzhou 510640, China

${ }^{c}$ State Key Laboratory of Infectious Disease Prevention and Control, National Institute for Communicable Disease Control and Prevention, Collaborative Innovation Center for Diagnosis and Treatment of Infectious Disease, Chinese Center for Disease Control and Prevention, Beijing, China 102206

$\dagger$ Electronic supplementary information (ESI) available. See DOI: 10.1039/c8ra01162a activating the phase II detoxifying/antioxidant enzymes, such as catalase (CAT), glutathione peroxidase (GSH-Px) and superoxide dismutase (SOD). ${ }^{6}$ Some studies have demonstrated that many dietary antioxidants could regulate oxidative stress in cells by activating Nrf2 or its related genes. ${ }^{7-10}$

As natural antioxidants, dietary peptides have attracted considerable attention due to their possible beneficial effects on human health. Increasing evidences have suggested that peptides isolated in variety of food protein, such as soy protein, ${ }^{11}$ hempseed protein ${ }^{\mathbf{1 2}}$ and egg white protein ${ }^{13}$ were able to inhibit oxidative stress not only by scavenging free radicals but also by increasing intracellular antioxidant enzyme activities and GSH levels. However, peptides are rarely reported in Chinese Baijiu, which is a natural solid-state distillated spirit from fermented grains (e.g., wheat, sorghum, corn, rice and glutinous rice), because they are often present in very low concentrations. ${ }^{\mathbf{1 4}}$ Although the antioxidant activities of peptides have been extensively studied up to now, to our best knowledge, there have been few reports concerning the identification of any antioxidant peptides from Chinese Baijiu.

In our previous study, a tripeptide (Pro-His-Pro, PHP, shown in Fig. 1A) was isolated and identified from Guojing and Gujinggong Baijiu, two famous types of Baijiu, and its antihypertensive activity was also studied. ${ }^{\mathbf{1 4}}$ The results indicated that PHP, as a noncompetitive inhibitor against angiotensin converting enzyme (ACE), possessed novel antihypertensive activity $\left(\mathrm{IC}_{50}=446 \mathrm{mM}\right)$ in vitro chemical assays. However, studies on the protective effects 
A<smiles>O=C(N[C@@H](Cc1c[nH]cn1)C(=O)N1CCC[C@H]1C(=O)O)[C@@H]1CCCN1</smiles>

C

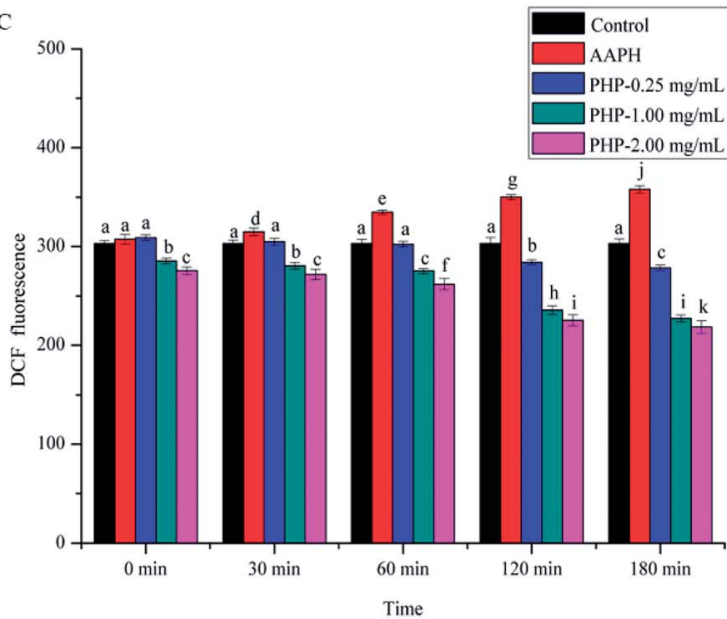

B
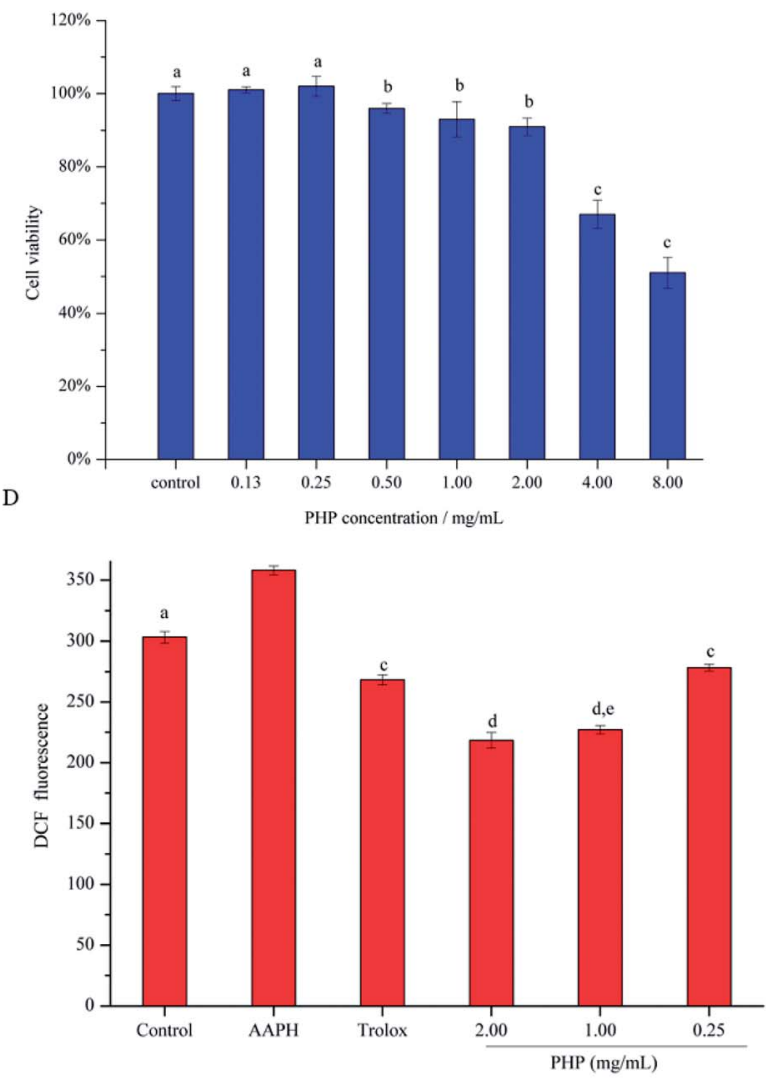

Fig. 1 The chemical structure of the tripeptide, Pro-His-Pro (PHP) (A). Effects of PHP on the viability of HepG2 cells (B). Intracellular ROS scavenging capacities of different concentrations of PHP and different incubation time under conditions of AAPH-induced oxidative stress in HepG2 cells (C). Intracellular ROS scavenging capacities of different concentrations of PHP and Trolox at 180 min of incubation (D). Data are presented as the mean \pm SD. Different letters indicate significant differences at $P<0.05, n=3$ data points.

of PHP against 2,2'-azobis (2-methylpropionamidine) dihydrochloride (AAPH)-induced oxidative stress in cells are still lacking.

Therefore, the aim of the present study was to determine the protective effects of PHP against oxidative stress induced by the potent oxidant (AAPH) in HepG2 cells, a useful model for evaluating the cytoprotective effect of natural antioxidants. The cell viability and several markers of oxidative damage were evaluated, such as the concentration of reduced GSH, GSSG and MDA, the generation of ROS, and the activities of antioxidant enzymes (CAT, GSH-Px and SOD). Furthermore, the gene and protein expression of the antioxidant enzymes, Nrf2 and Keap1 were determined to validate if PHP modulated the antioxidant enzymes via Nrf2 signaling in HepG2 cells.

\section{Material and methods}

\subsection{Chemicals}

2,2'-Azobis (2-methylpropionamidine) dihydrochloride (AAPH) and 6-hydroxy-2,5,7,8-tetramethylchroman-2-carboxylic acid (Trolox) were purchased from Sigma-Aldrich (St. Louis, MO, USA). The synthetic peptide, PHP (Fig. 1A), with 99\% purity, was purchased from GL Biochem Ltd. (Shanghai, China). Microscale MDA, SOD, CAT, GSH-Px, and bicinchoninic acid (BCA) protein assay kits were purchased from Nanjing Jiancheng Institute of
Biotechnology (Nanjing, China). ROS and GSH/GSSG assay kits were purchased from Beyotime Institute of Biotechnology (Shanghai, China). Cell counting kit (CCK-8) assay was obtained from Dojindo (Kumamoto, Japan). Trizol reagent was from Life Technologies (USA). Anti-CAT (GTX110704) was purchased from GenTex Inc. (USA). Anti-SOD (Ab13533), anti-GSH-Px (Ab22604), anti-Nrf2 (Ab31163) and anti-Keap1 (Ab218815) were purchased from Abcam (Cambridge, UK). Anti-glyceraldehyde-3-phosphate dehydrogenase (GAPDH, AB-P-R001) was from JK GREEN Company (Beijing, China).

\subsection{Cell culture}

Human hepatoma HepG2 cells were obtained from China Union Medical University (Beijing, China). These cells were grown in Dulbecco's modified Eagle's medium (DMEM) medium from Corning (New York, America) in a humidified incubator containing $5 \% \mathrm{CO}_{2}$ and $95 \%$ air at $37{ }^{\circ} \mathrm{C}$, supplemented with $10 \%$ Corning fetal bovine serum (FBS) and $50 \mu \mathrm{g}$ $\mathrm{mL}^{-1}$ of each of the antibiotics gentamicin, penicillin, and streptomycin (all from Sigma-Aldrich, USA). The culture medium was changed every two days and was split at $80 \%$ to $90 \%$ confluency using $0.25 \%$ trypsin and $0.02 \%$ EDTA solution. 


\subsection{Evaluation of HepG2 cell viability treated by PHP}

To avoid the mortality produced by PHP, suitable concentrations of the test samples were determined. HepG2 cells were plated in Costa 96-well plates (Corning, NY, America) at densities of $1 \times 10^{5}$ cells per mL. Cells expect the controls were treated with $10 \mu \mathrm{L}$ of fresh DMEM containing different concentrations of PHP (0.13, $0.25,0.50,1.00,2.00,4.00$ and $8.00 \mathrm{mg} \mathrm{mL}^{-1}$ ) for $24 \mathrm{~h}$ in the humidified incubator containing $5 \% \mathrm{CO}_{2}$ and $95 \%$ air at $37{ }^{\circ} \mathrm{C}$. The cell viability assays were conducted using a non-radioactive CCK-8 (cell counting kit-8) method. ${ }^{15,16}$ Then the plates were washed twice with PBS and treated with $10 \mu \mathrm{L}$ of CCK-8 solution for $4 \mathrm{~h}$. The absorbance of each well in the plates was recorded at $450 \mathrm{~nm}$ in a Synergy H1multimode plate reader (BioTek, America).

\subsection{Protective effects of PHP on ROS generation against AAPH-induced oxidative stress in HepG2 cells}

Cellular oxidative stress due to AAPH-induced ROS was measured by the dichlorofluorescin-diacetate (DCFH-DA) assay. ${ }^{17}$ DCFH is oxidized to dichlorofluorescein (DCF), which fluoresces, by intracellular oxidants. ${ }^{18}$ To determine the level of intracellular ROS, the intensity of the fluorescence was read at 485 (excitation) and 525 (emission) $\mathrm{nm}$ by a microplate reader (BioTek, America) as described by Zhang with minor modifications. ${ }^{6}$ A condition of cellular oxidative stress is evoked when HepG2 cells are treated with $200 \mu \mathrm{M}$ AAPH according to the previous reports. ${ }^{19}$ HepG 2 cells were seeded in 96-well plates at densities of $1 \times 10^{5}$ cells per $\mathrm{mL}$ and treated with DMEM (control group) or DMEM containing different concentrations of PHP $\left(0.25,1.00\right.$ and $\left.2.00 \mathrm{mg} \mathrm{mL}^{-1}\right)$ or $1.00 \mathrm{mg} \mathrm{mL}^{-1}$ of Trolox (for positive control group) before incubation with $10 \mu \mathrm{M}$ of DCFH-DA for $0.5 \mathrm{~h}$ at $37^{\circ} \mathrm{C}$. The cells were washed three times with PBS and treated with $200 \mu \mathrm{M}$ AAPH or DMEM (control group) for $3 \mathrm{~h}$. Aliquots of the final reaction mixture were evaluated after incubation periods of $0,30,60,120$ and $180 \mathrm{~min}$ at $37^{\circ} \mathrm{C}$. The fluorescence intensity was read at 485 (excitation) and 525 (emission) nm. An ROS assay kit with DCFH-DA as a fluorescent probe was used to determine the levels of intracellular ROS.

\subsection{Determination of MDA and antioxidant enzymes activities}

HepG2 cells were plated in Costa 24 -well plates at concentrations of $1 \times 10^{5}$ cells per mL. Cells were treated with different concentrations of PHP $\left(0.25,1.00\right.$ and $\left.2.00 \mathrm{mg} \mathrm{mL}^{-1}\right)$ or DMEM (control group) or $1.00 \mathrm{mg} \mathrm{mL} \mathrm{m}^{-1}$ of Trolox (for positive control group) for $2 \mathrm{~h}$ and were then washed twice with PBS. To induce oxidative stress, the cells were treated with $200 \mu \mathrm{M}$ AAPH for $3 \mathrm{~h}$. The cells were washed three times with PBS and then lysed with RIPA lysis buffer (Cwbio, Beijing, China) containing $1 \mathrm{mM}$ PMSF (Cwbio, Beijing, China) at $4{ }^{\circ} \mathrm{C}$ for $10 \mathrm{~min}$. The treated cells were then collected and centrifuged at $14000 \times g$ for $5 \mathrm{~min}$ to obtain the supernatants. MDA contents and the activities of SOD, CAT, and GSH-Px in the supernatants were determined using commercially available enzyme assay kits. The cytosolic protein concentration was quantified by a commercially BCA kit with bovine serum albumin (BSA) as the standard.

\subsection{Measurement of oxidized glutathione (GSSG) and glutathione (GSH)}

HepG2 cells were plated in Costa 24 -well plates at concentrations of $1 \times 10^{5}$ cells per $\mathrm{mL}$ and then treated with samples by the same method that was described in the section on the Determination of MDA and antioxidant enzymes activities. The cells were digested with $0.25 \%$ trypsin-EDTA at $37^{\circ} \mathrm{C}$ for $5 \mathrm{~min}$ followed by washing with PBS. Afterwards, the serumcontaining DMEM was added to terminate the digestion. Then, the mixture was centrifuged at $14000 \times g$ for $10 \mathrm{~min}$ at $4{ }^{\circ} \mathrm{C}$ to collect the cell debris pellets. After removing intracellular proteins, the treated cell debris pellets were rapidly cycled between $-80{ }^{\circ} \mathrm{C}$ and $37{ }^{\circ} \mathrm{C}$ twice. Then, the cell debris pellets were kept at $4{ }^{\circ} \mathrm{C}$ for $5 \mathrm{~min}$, and the supernatants were obtained after centrifugation at $1000 \times g$ for $10 \mathrm{~min}$. The cell supernatants were used to measure the contents of GSSG and GSH according to a GSH/GSSG assay kit.

\subsection{Real-time PCR}

Total RNA of HepG2 cells were extracted using TRIzol reagent (Life Technologies, USA) according to the directions of manufacturer. Reverse transcription of $1 \mu \mathrm{g}$ RNA was conducted with M-MLV Reverse Transcriptase kit (Promega Corporation, USA) to generate cDNAs for qPCR detection. The SYBR-Green TwoStep RT-qPCR assay was performed in an ABI 7500 real-time PCR system using the Applied Biosystems Master Mix reagents kit. The RT-qPCR was performed using the following amplification profile: 1 cycle at $95{ }^{\circ} \mathrm{C}$ for $10 \mathrm{~min}, 40$ cycles at $95{ }^{\circ} \mathrm{C}$ for $10 \mathrm{~s}$ and $60{ }^{\circ} \mathrm{C}$ for $1 \mathrm{~min}$. All samples were assayed in triplicate and the data were analyzed by the $\Delta \Delta \mathrm{CT}$ method. ${ }^{20}$ GAPDH primers were added as the internal control. Primers used in this assay were listed in Table 1.

\subsection{Protein determination by western blotting}

HepG2 cells were lysed by ice-cold RIPA lysis buffer with Complete Protease Inhibitor Cocktail Tablets (Roche), and incubated on ice for $30 \mathrm{~min}$. The lysates were collected by centrifugation at $4{ }^{\circ} \mathrm{C}$ at $14000 \times g$ for $15 \mathrm{~min}$ to obtain the supernatants. Then, the supernatants were boiled in $5 \times$

Table 1 Primer sequences

\begin{tabular}{|c|c|c|c|}
\hline Gene & $\begin{array}{l}\text { Product size } \\
\text { (bp) }\end{array}$ & Primer & $5^{\prime}$ primer sequences $3^{\prime}$ \\
\hline \multirow[t]{2}{*}{ GAPDH } & 185 & Forward & GGTGGTCTCCTCTGACTTCAACA \\
\hline & & Reverse & GTTGCTGTAGCCAAATTCGTTGC \\
\hline \multirow[t]{2}{*}{ SOD } & 115 & Forward & TGGAGATAATACAGCAGGCT \\
\hline & & Reverse & AGTCACATTGCCCAAGTCTC \\
\hline \multirow[t]{2}{*}{ CAT } & 97 & Forward & CCTTCGACCCAAGCAA \\
\hline & & Reverse & CGATGGCGGTGAGTGT \\
\hline \multirow[t]{2}{*}{ GSH-Px } & 172 & Forward & AGAAGTGCGAGGTGAACGGT \\
\hline & & Reverse & CCCACCAGGAACTTCTCAAA \\
\hline \multirow[t]{2}{*}{ Nrf2 } & 172 & Forward & AGTGTGGAGAGGTATGAGCC \\
\hline & & Reverse & CGTTCCTCTCTGGGTAGTAA \\
\hline \multirow[t]{2}{*}{ Keap1 } & 126 & Forward & AGAGCGGGATGAGTGGCA \\
\hline & & Reverse & GCTGAATTAAGGCGGTTTGTC \\
\hline
\end{tabular}


Laemmli buffer (Sigma-Aldrich, USA) for $10 \mathrm{~min}$ followed by electrophoresed in $10 \%$ sodium dodecyl sulfate polyacrylamide gel (SDS-PAGE). Proteins were transferred onto a NC membrane (Millipore, USA) and blocked with 5\% skim milk for $1 \mathrm{~h}$ at room temperature. After being washed 5 times in tris-buffered saline with Tween 20 (TBST), the membranes were incubated with indicated primary antibodies at 1:1000 dilutions overnight at $4{ }^{\circ} \mathrm{C}$. Afterwards, the membranes were washed 5 times again with TBST and continued to be incubated with peroxidaseconjugated secondary antibodies at $1: 5000$ dilutions for $1 \mathrm{~h}$ at room temperature. After the membranes were washed, the proteins were detected using ECL (Millipore, USA) according to the protocol by Yeh and Yen. ${ }^{21}$ GAPDH was used as a loading control.

\subsection{Statistical analysis}

All experiments were repeated for three times and the results were expressed as the mean \pm standard deviation (SD). All data were analyzed by one-way analysis of variance (ANOVA) using SPSS (version 16.0, IBM Inc., New York, USA). Pearson correlation coefficients were calculated by SPSS (version 16.0, IBM Inc., New York, USA). Significant differences $(P<0.05)$ were determined using the LSD range test.

\section{Results and discussion}

\subsection{Effect of PHP on cell viability}

Cell viability is often used as an indicator of cytotoxicity. Prior to investigate the antioxidant activity of PHP, the toxic effect of PHP on HepG 2 cells was detected by CCK- 8 assay. As shown in Fig. 1B, after being treated with PHP for $24 \mathrm{~h}$, the cell viability had a concentration-dependent decrease with increasing PHP concentrations, thus, high concentrations of PHP treatment had obvious cytotoxic effects on HepG2 cells. The cell viability decreased significantly $(P<0.05)$ when the concentration of PHP $\geq 4.00 \mathrm{mg} \mathrm{mL}^{-1}$, indicating severe damages in HepG2 cells. Therefore, the appropriate concentration of PHP was set at $2.00 \mathrm{mg} \mathrm{mL}^{-1}$, suggesting that PHP $\leq 2.00 \mathrm{mg} \mathrm{mL}^{-1}$ exhibited negligible toxicity on HepG2 cells. In addition, another two experiments were also set up using concentrations of 0.25 and $1.00 \mathrm{mg} \mathrm{mL}{ }^{-1}$ to further investigate the protective effects of different concentrations of the samples.

\subsection{Preventive effects of PHP on ROS generation}

To analyze the cytoprotective effects of PHP against AAPHinduced oxidative stress in HepG2 cells, the levels of ROS were determined, and the treatment concentrations and incubation time of PHP samples were taken into consideration. As shown in Fig. 1C, compared with the control group, the ROS level in the AAPH group increased from 30 to $180 \mathrm{~min}$, which indicated enhanced oxidative stress. However, pretreatment with different concentrations of PHP caused the ROS levels to decrease when compared to those in the AAPH group from 30 to $180 \mathrm{~min}$. The ROS levels for the low-level treatment group were $3.5-22.3 \%$, for the middle-level treatment group they were 11.1$36.6 \%$, and for the high-level treatment group they were 14.0-
$39.1 \%$ from 30 to $180 \mathrm{~min}$. Also noted was that there were negative correlations between ROS generation and PHP concentration $(r=-0.891, P=0.299)$ or incubation time when the cells were treated with $0.25 \mathrm{mg} \mathrm{mL}^{-1}(r=-0.980, P=0.003)$, $1.00 \mathrm{mg} \mathrm{mL}^{-1}(r=-0.967, P=0.007)$ and $2.00 \mathrm{mg} \mathrm{mL}^{-1}(r=$ $-0.969, P=0.006)$ of PHP. These findings indicated that the levels of ROS decreased in a concentration- and time-dependent manner, similar to the reports by $\mathrm{Zhao}^{22}$ and $\mathrm{Wu}^{19}$.

As shown in Fig. 1D, Trolox was used as the positive standard in this study to deeply evaluate the antioxidant of PHP. After treating with $1.00 \mathrm{mg} \mathrm{mL}{ }^{-1}$ of Trolox for $2 \mathrm{~h}$, the intracellular ROS level in the positive control group $(268.3 \pm 4.18)$ significantly $(P<0.05)$ decreased comparing with AAPH group, proving that Trolox was a good antioxidant in the HepG2 model. But the levels of ROS in Trolox group were respectively $15.3 \%$ and $18.5 \%$ higher than the PHP treatment groups (1.00 and $2.00 \mathrm{mg} \mathrm{mL}^{-1}$ ) when the incubation time was at $180 \mathrm{~min}$, which revealed that PHP showed stronger ROS scavenging ability than Trolox in a certain concentration range $\left(\geq 1.00 \mathrm{mg} \mathrm{mL}^{-1}\right)$. Pretreatment with 1.00 and $2.00 \mathrm{mg} \mathrm{mL}{ }^{-1}$ of PHP significantly $(P<0.05)$ decreased ROS generation and reduced the levels of ROS to those of the control untreated cells even at the beginning of the reaction, which indicated that the treated cells were partly protected by PHP against oxidative damage. Comparable results had been reported by Wang, ${ }^{23}$ Agrawal $^{24}$ and $\mathrm{Du}^{25}$, stating that the antioxidant peptides derived from grains (e.g. corn, millet and oat) could also protect HepG2 cells from oxidative damage by scavenging ROS.

\subsection{Activities of CAT, SOD and GSH-Px}

It is well-documented that antioxidants have direct and indirect cellular antioxidant capacities. ${ }^{\mathbf{2 6}}$ The direct antioxidant capacity is the ability of the antioxidant itself to scavenge ROS via donation of a hydrogen or electrons, while the indirect antioxidant capacity is the ability of an antioxidant to prevent oxidative stress by inducing the expression of the antioxidant enzymes and antioxidant genes. Reportedly, ${ }^{27,28}$ the intracellular enzyme system, CAT converts hydrogen peroxide into oxygen and water, and SOD catalyzes the conversion of highly reactive superoxide anion to less reactive oxygen and hydrogen peroxide through disproportionation reaction. Excess hydrogen peroxide can be reduced by CAT and GSH-Px to prevent hydrogen peroxide from being converted into more active species, such as hydroxyl radicals, which promote cell death. Thus, changes in the activities of antioxidant enzymes can be considered biomarkers of the antioxidant response. In this study, both antioxidant mechanisms were evaluated, including nonenzymatic and enzymatic antioxidant systems.

As shown in Fig. 2A-C, treatment with AAPH for $3 \mathrm{~h}$ could significantly $(P<0.05)$ suppress CAT activity $(72.2 \%)$, GSH-Px activity $(31.9 \%)$ and SOD activity $(50.2 \%)$ compared to the control group, which demonstrated that AAPH could increase oxidative stress in HepG2 cells, similar to the report by Zhao. ${ }^{22}$ However, the CAT, GSH-Px and SOD activities significantly $(P<$ 0.05 ) increased with $0.25 \mathrm{mg} \mathrm{mL}^{-1}$ (12.6 times, $95.2 \%$ and $27.5 \%$, respectively), $1.00 \mathrm{mg} \mathrm{mL}^{-1}$ (13.8 times, 1.0 times, and 

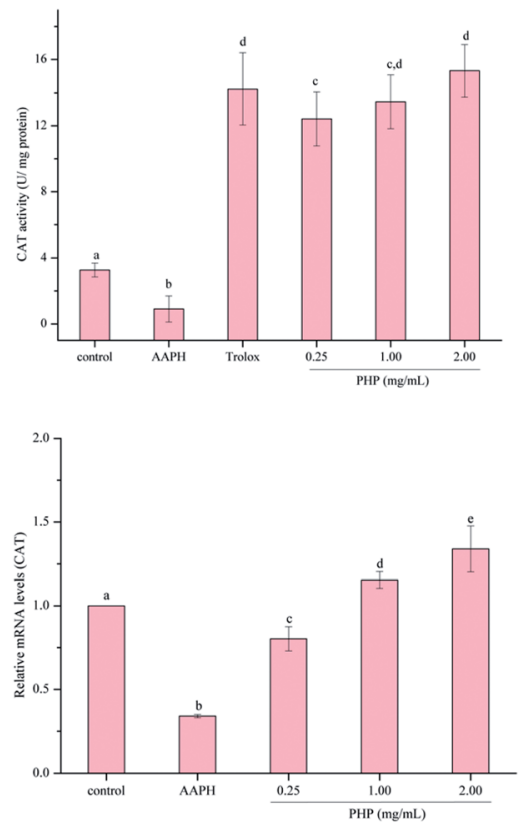

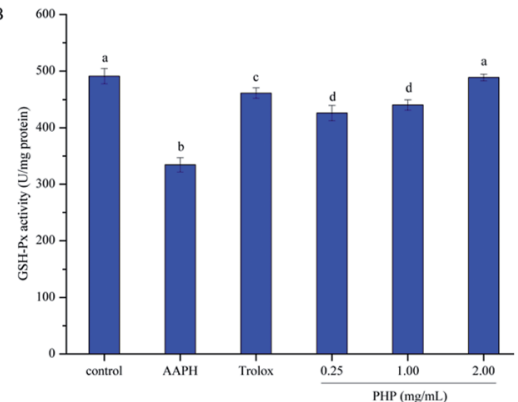

E

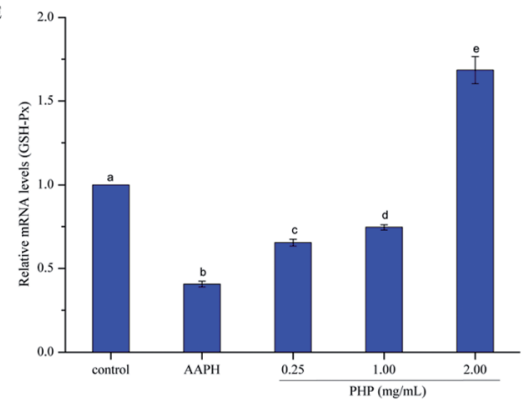

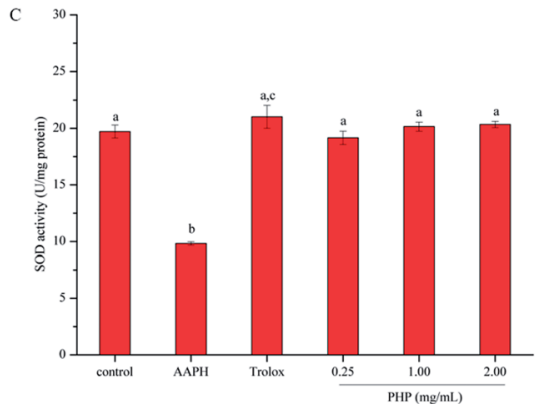

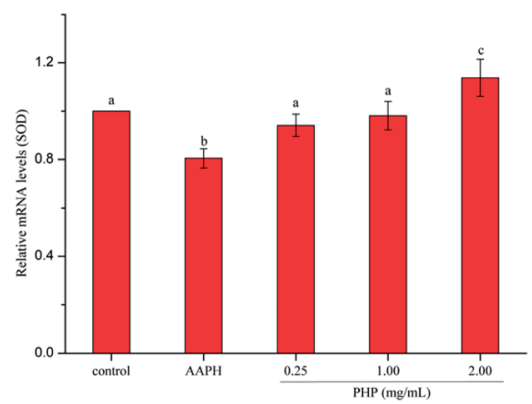

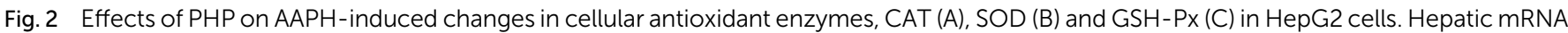

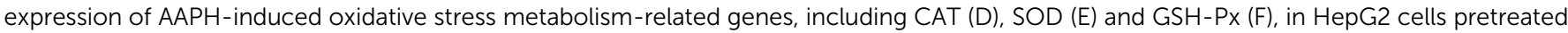

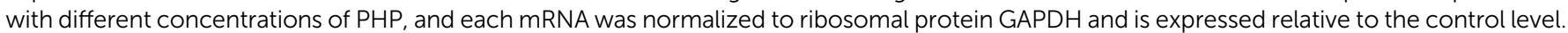

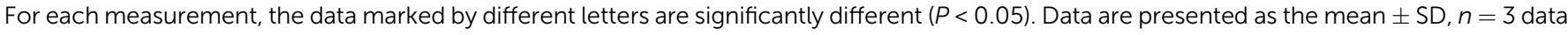
points.

$31.7 \%$, respectively) and $2.00 \mathrm{mg} \mathrm{mL}^{-1}$ (15.8 times, 1.1 times and $46.1 \%$, respectively) of PHP pretreatment compared with those in the AAPH group. These results indicated that PHP showed preventive effects against AAPH-induced changes in the activities of CAT, GSH-Px and SOD. Moreover, the higher the concentration of PHP, the better the activities of the antioxidant enzymes, which was consistent with the report by Zhang. ${ }^{15}$ In addition, the activity of SOD was positively correlated with the activities of CAT and GSH-Px $(r=0.858, P=0.343$ and $r=0.780$, $P=0.431$ ), similar to the previous reports. ${ }^{6,15}$ Wang, L. et al. ${ }^{23}$ also observed that the Gly-Leu-Leu-Leu-Pro-His (GLLLPH) identified from corn gluten peptides fractions increased the activity levels of SOD (from $86.54 \%$ to $114.14 \%$ ), CAT (from $71.91 \%$ to $107.64 \%$ ) and GSH-Px (from $70.52 \%$ to $103.01 \%$ ). GLLLPH contained Pro-His, therefore, the peptide bond or the peptide conformation might have enhanced the antioxidant activity of PHP.

It was noteworthy that there was no significant difference on the activity of CAT, GSH-Px and SOD between the Trolox group and high-level treatment of PHP groups. However, the antioxidant enzymes activities in the middle-level of PHP group were all lower than that of Trolox group even though the samples were at the same concentration. Previous studies have demonstrated that peptides (i.e. oat proteins derived peptides, phosphopeptides from hen egg yolk phosvitin and corn gluten peptides) possessed antioxidant capacities and protected cells from oxidative stress-related damage by improving the activities of important intracellular antioxidant enzymes even more powerful than the positive control (Trolox)..$^{23,25,29}$ In this study, pretreatment with PHP also effectively enhanced the activities of the antioxidant defense systems of the HepG2 cells as indicated by the increased CAT, SOD and GSH-Px levels.

\subsection{Reduced GSH, GSSG and total GSH concentration}

GSH is the main non-enzymatic antioxidant defense within the cell. Previous studies have proven that GSH-Px catalyzes the conversion of glutathione from the reduced form GSH to the oxidized form GSSH, and the depletion of GSH and the generation of GSSG reflect the degree of intracellular oxidation. ${ }^{23,30,31}$ In contrast, an increase in GSH concentration can be expected as the cell protect itself against potential oxidative damage. Thus, the contents of GSH and GSSG were both determined in this study. As shown in Fig. 3A and B, AAPH group exhibited a dramatic decrease in cytoplasmic GSH and increase in GSSG, which indicated oxidation in the HepG2 cells. Compared with the levels of GSH and GSSG in the AAPH group, these levels in the PHP groups were significantly $(P<0.05)$ increased by $30.6-76.4 \%$ and decreased by 25.1-86.9\%, respectively. Significant differences was obtained between the Trolox group $(10.67 \pm 0.83 \mu \mathrm{M})$ and high-level treatment group $(12.26 \pm 1.40 \mu \mathrm{M})$, which revealed that PHP were probably more effective than Trolox. These results showed that PHP could effectively promote the non-enzymatic antioxidant defense of HepG2 cells in a certain concentration range. Meanwhile, the GSH content in the control group $(12.92 \pm 0.78 \mu \mathrm{M})$ was higher than that in the group treated with a high concentration of PHP $(11.08 \pm 0.97 \mu \mathrm{M})$. However, no significant difference in the GSSG level was obtained between the control group $(0.33 \pm 0.02$ $\mu \mathrm{M})$ and the high-level treatment group $(0.30 \pm 0.04 \mu \mathrm{M})$. There was no significant difference in the total GSH levels (GSH + GSSG) among all of the groups. These results revealed that the levels of 

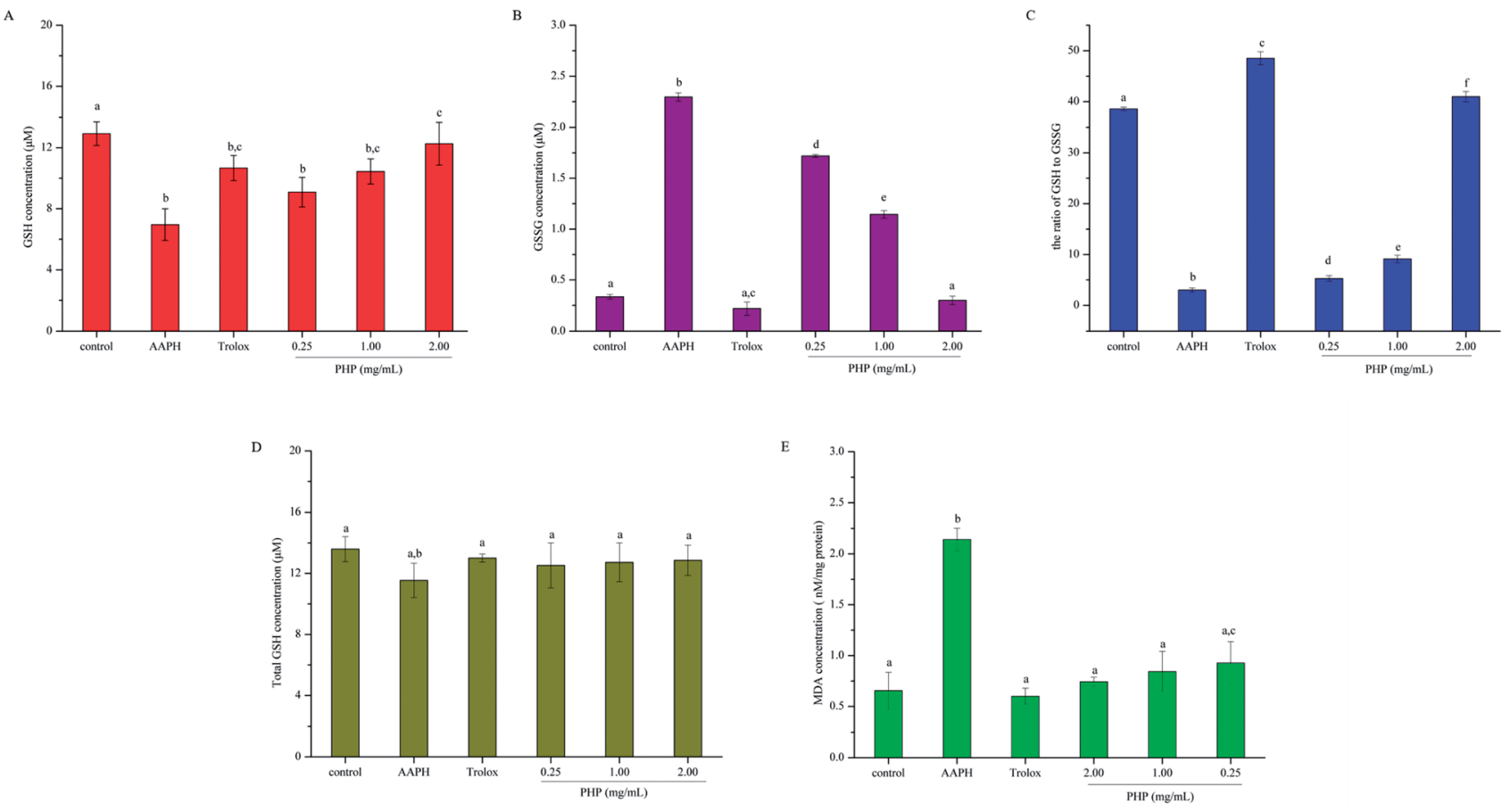

Fig. 3 Effects of different concentrations of PHP on AAPH-induced changes in intracellular reduced GSH (A), GSSG (B), the ratio of GSH to GSSG (C), total GSH (D) and MDA (E) in HepG2 cells. Values are the mean $\pm S D, n=3$ data points. Different letters indicate statistically significant differences $(P<0.05)$ among different groups.

total GSH in the cells were stable even during periods of oxidative stress (Fig. 3D), and was consistent with the results reported by Wang..$^{23}$

To deeply understand the effect of PHP on AAPH-induced changes in the levels of GSH and GSSG in HepG2 cells, the ratio of GSH to GSSG was also assessed. As shown in Fig. 3C, the lowest ratio of GSH to GSSG was obtained in the AAPH group $(3.02 \pm 0.39)$. A significant difference $(P<0.05)$ was even found between the low-level treatment group of PHP and the AAPH group, which indicated that the AAPH group had high level of oxidative stress and the stress was overcome by PHP in a certain concentration range. The ratio of GSH to GSSG changed in a concentration-dependent manner, and it was remarkable that the Trolox group exhibited the highest ratio of GSH to GSSG $(48.50 \pm 1.27)$, followed by the high-level treatment of PHP group (40.96 \pm 1.48$)$. Even so, pretreatment with PHP could prevent GSH from decreasing and GSSG from increasing below steady-state levels in the presence of AAPH, and so helped the cells to inhibit oxidative damage, which provided the visual evidences for the preventive effect of PHP against AAPHinduced oxidative stress. The results were accordance with several previous studies, ${ }^{25,29}$ which suggested that the increased generation of GSSG and the decreased level of GSH could be suppressed by the antioxidant peptides.

\subsection{MDA levels}

An important step in the degradation of cell membranes is the reactions of ROS with the double bonds of polyunsaturated fatty acids (PUFAs) to yield lipid hydroperoxides. During the degradation, a series of aldehydes can be formed, and MDA is one of the main products of lipid hydroperoxides. ${ }^{32}$ As a biomarker for lipid peroxidation, the cytoplasmic concentration of MDA was measured in HepG2 cells. As shown in Fig. 3E, the AAPH group evoked a significant increase (2.3 times) in the cellular concentration of MDA than that in the control group. However, pretreatment with all tested concentrations of PHP for $2 \mathrm{~h}$ prevented the increases in MDA contents by $56.7-65.2 \%$. This evidence showed the level of AAPH-induced lipid peroxidation was lower in these cells incubated with PHP for $2 \mathrm{~h}$, even if the concentration of PHP was as low as $0.25 \mathrm{mg} \mathrm{mL}^{-1}$ (Fig. 3E). PHP could markedly attenuated the generation of MDA even more effectively than Trolox when they were in the same level even though no significant difference were found among the groups. Therefore, after pretreatment with different concentrations of PHP, the levels of MDA tended to be lower than those of the control untreated cells even after $3 \mathrm{~h}$ of treatment with $200 \mu \mathrm{M}$ AAPH. Similar findings were obtained from the corn peptides, which significantly reduced the increase of MDA contents. ${ }^{9}$

\subsection{Gene expression of antioxidant enzymes, Nrf2 and Keap1}

Activation of Nrf2 and its downstream phase II detoxifying/ antioxidant enzymes are considered to have therapeutic potential in preventing diseases associated with oxidative stress and inflammation. ${ }^{33}$ PHP was shown to possess antioxidant activities in this study, thus, we hypothesized that PHP suppressed AAPH-induced oxidative stress through activation of Nrf2 and the phase II detoxifying/antioxidant enzymes (CAT, SOD, and GSH-Px). To test this, HepG2 cells were exposed to a range concentrations of PHP for $4 \mathrm{~h}$ followed by treatment 
with AAPH for $3 \mathrm{~h}$, and the gene transcriptions of SOD, CAT, GSH-Px, Nrf2 and Keap1 were examined by RT-PCR.

As shown in Fig. 2D-F, the groups treated with AAPH alone showed the lowest mRNA levels of CAT, SOD and GSH-Px, indicating that AAPH increased oxidative stress. However, pretreatment with different concentrations of PHP could significantly $(P<0.05)$ up-regulated the gene expressions of these antioxidant enzymes variously in an obvious concentration-dependent manner, which was similar to the previous reports on antioxidants. ${ }^{15,34}$ Particularly, compared with the control group, the cells treated with 1.00 and $2.00 \mathrm{mg}$ $\mathrm{mL}^{-1}$ PHP showed significant increase in mRNA levels of CAT, which was consistent with the activity of CAT, indicating that PHP had the similar concentration-dependent manner effects on the gene expression of CAT (Fig. 2D). It was also remarkable that the mRNA levels of SOD and GSH-Px in the high-level treatment groups $(1.14 \pm 0.07$ and $1.69 \pm 0.08$, respectively) were relative higher than those in the control group, while pretreatment with $\mathrm{PHP}<2.00 \mathrm{mg} \mathrm{mL}^{-1}$ made very little effects on the mRNA levels of SOD and GSH-Px (Fig. 4E and F). These results suggested that $\mathrm{PHP}$ exhibited novel antioxidant activities by up-regulating the expression of antioxidant enzymes in
HepG2 cells in a certain concentration range $\left(\geq 2.00 \mathrm{mg} \mathrm{mL}^{-1}\right)$, similar to the previous reports. ${ }^{30,35}$ Additionally, there were positive correlations between the mRNA expression levels and activities of CAT ( $r=0.942, P=0.218)$, SOD $(r=0.763, P=$ $0.448)$ and GSH-Px $(r=0.990, P=0.089)$. Furthermore, the mRNA level of SOD was positively correlated with the mRNA levels of CAT and GSH-Px $(r=0.874, P=0.323$ and $r=0.993, P$ $=0.072$ ), which was consistent with the results in the activities of CAT, SOD and GSH-Px assay and the reports by Zhang et al. ${ }^{6,15}$

Meanwhile, the PHP treatment could alter the mRNA expression levels of Nrf2 and Keap1 (Fig. 4A and B). The lowest mRNA level of Nrf2 $(0.31 \pm 0.02)$ and the highest mRNA level of Keap1 $(1.72 \pm 0.03)$ were in the AAPH groups without peptide treatment. It was noteworthy that the gene expression levels of Nrf2 in PHP treatment groups $\left(\geq 1.00 \mathrm{mg} \mathrm{mL}^{-1}\right)$ were respectively 1.2- to 1.3-times higher than that of the control group, and this was comparable to that of the feruloyl oligosaccharides (FOs) from wheat bran increased the gene expression level of Nrf2. ${ }^{6}$ Whereas pretreatment with a range concentrations of PHP down-regulated the expression level of Keap1, demonstrating that PHP could cause a dissociation of Nrf2/Keap1 complex, similar to the previous report. ${ }^{36}$ It was usually
A

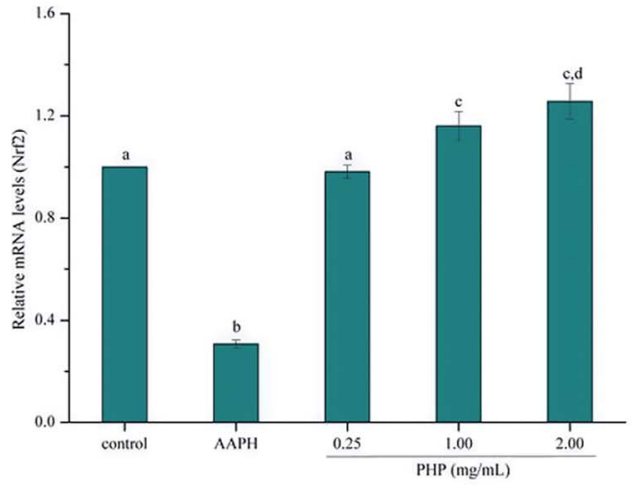

C

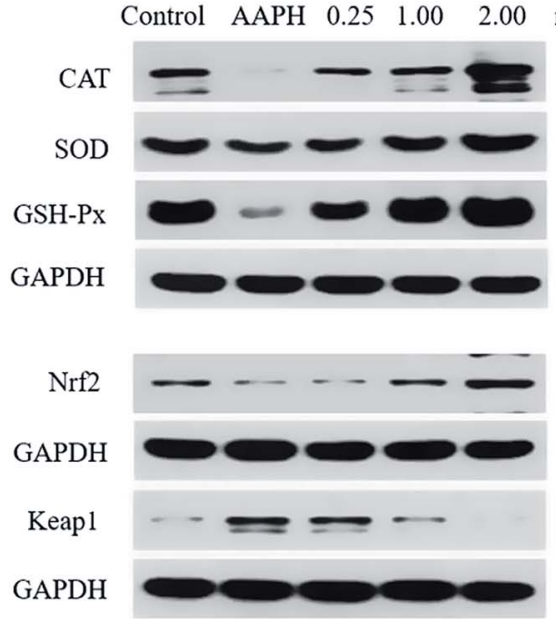

B

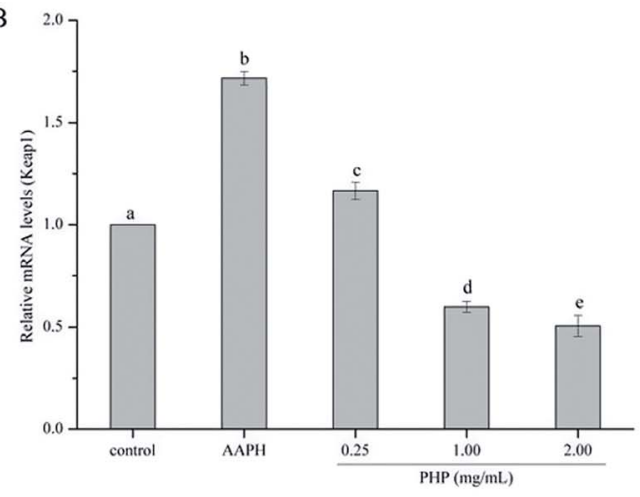

D

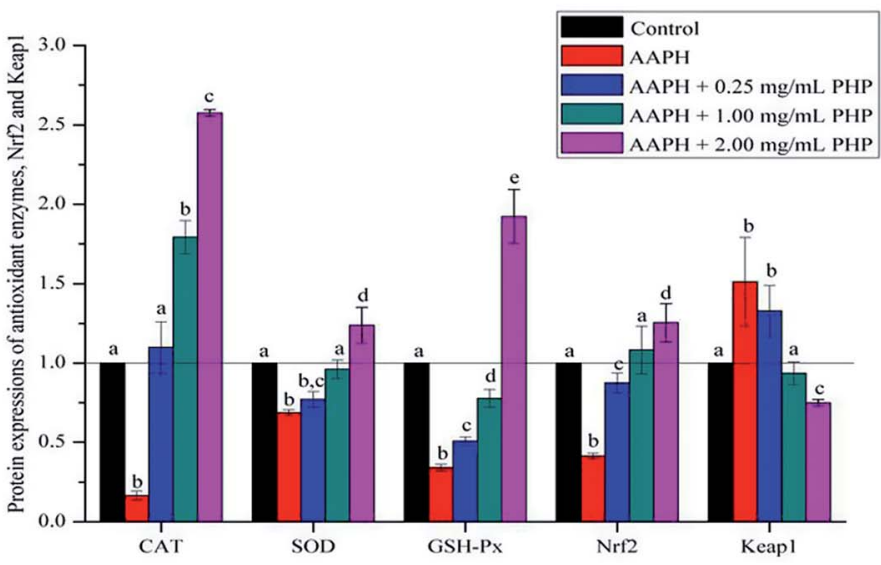

Fig. 4 Hepatic mRNA expression of antioxidant signaling pathway related genes including nuclear factor erythroid 2-related factor 2 (Nrf2) (A) and Kelch-like ECH-associated protein-1 (Keap1) (B) in HepG2 cells pretreated with different concentrations of PHP, and each mRNA was normalized to ribosomal protein GAPDH and is expressed relative to the control level. Hepatic protein expression of oxidative stress metabolismrelated protein and antioxidant signaling pathway related protein ( $C$ and $D)$ in HepG2 cells, pretreated with different concentrations of PHP, and each protein expression was normalized to ribosomal protein GAPDH and is expressed relative to the control level. Data are expressed as the mean $\pm \mathrm{SD}$. Different letters indicate significant difference at $P<0.05$. 


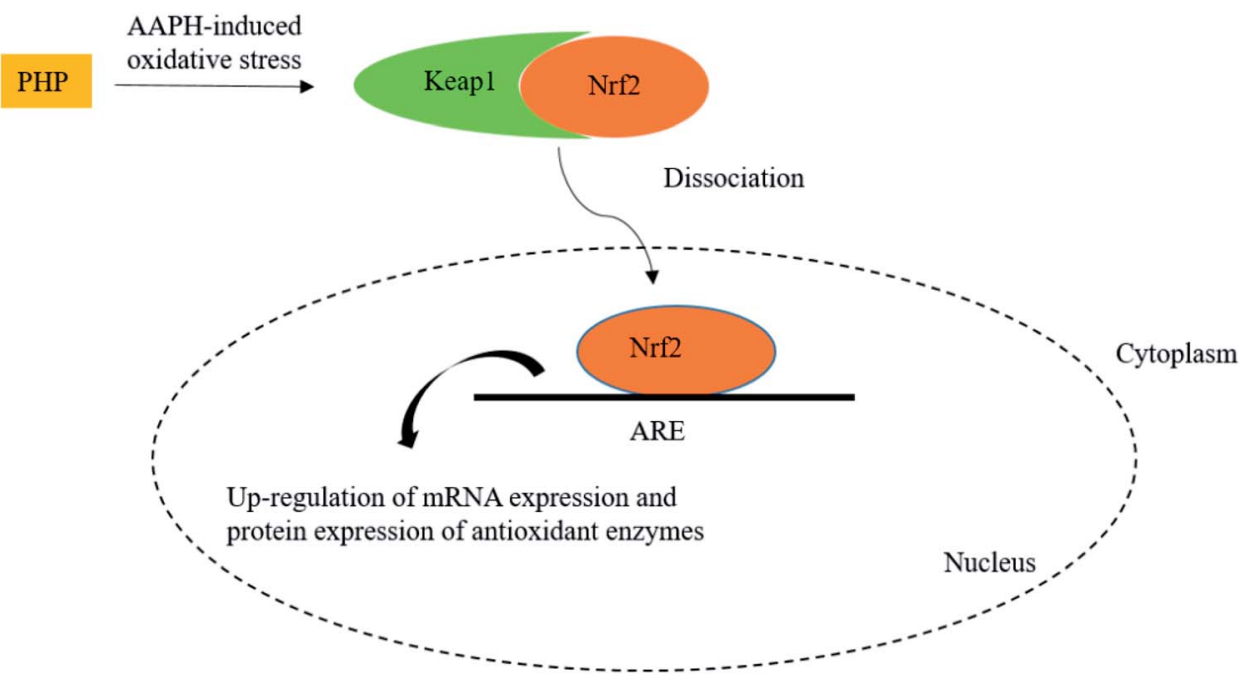

Fig. 5 Scheme summarizing the inhibition of AAPH-induced oxidant injury via the up-regulation of antioxidant enzymes via Nrf2/ARE pathway.

considered that antioxidant peptides could decrease the affinity of Nrf2 and Keap1, which was consistent with this study.

\subsection{Protein expression of antioxidant enzymes, Nrf2 and Keap1}

As a sensitive receptor for oxidative stress, Nrf2/Keap1 signaling pathway plays a crucial role in preventing cells from apoptosis, stress, inflammation and tumor, which is the most important intrinsic antioxidant stress pathway yet discovered. Previous studies have proven that antioxidants can regulate oxidative stress in cells by activating Nrf2 or its related genes. ${ }^{36,37}$ The samples with the concentrations of PHP $\geq 0.25 \mathrm{mg} \mathrm{mL}^{-1}$ have been testified to possess strong antioxidant ability and thus were further analyzed in this study. In order to identify whether the Nrf2 expression was modulated by PHP, an immunoblotting (western blotting) analysis was performed.

It is not surprising that AAPH treatment alone decreased the expression levels of CAT, SOD and GSH-Px and was similar to the mRNA levels and the enzymes activities compared with the control groups, which indicated that AAPH-induced antioxidant enzymes decreased by increasing ROS generation (Fig. 4C and D). However, the CAT, SOD, and GSH-Px protein expression levels in the PHP treatment groups were 6.6-15.5, 1.1-1.8 and 1.5-5.7 times higher than the AAPH group, suggesting that PHP treatment up-regulated the mRNA and protein expression levels of CAT, SOD and GSH-Px at the same time, and this was consistent with a previous study. ${ }^{32}$ Additionally, positive correlations between the mRNA and protein expression levels of CAT, SOD and GSH-Px $(r=0.977 \& P=0.135, r=0.976 \& P=0.141$ and $r=0.995 \& P=0.064)$ were obtained.

Meanwhile, the results showed that the Nrf2 protein expression in the PHP treatment group increased by 2.1-3.0 times while the Keap1 expression level was decreased by 0.5-0.9 time than the AAPH treated group (Fig. 4C and D), which demonstrated that PHP could decrease the affinity of Nrf2 for Keap1, similar to the reports by Balogun ${ }^{38}$ and $\mathrm{Ye}^{39}$. PHP might stimulate Nrf2-mediated ARE activation by enhancing Nrf2 protein level and reducing Keap1 at the same time, in other words, by increasing the ratio of Nrf2/Keap1 according to Tanigawa. ${ }^{36}$ Additionally, the Nrf2 protein expression positively correlated with the CAT, SOD and GSH-Px protein levels $(r=$ $0.996 \& P=0.057, r=0.987 \& P=0.103$ and $r=0.922 \& P=$ 0.253). These observations suggested that an accumulation of Nrf2 protein might contribute to the induction of ARE-mediated antioxidant gene expression after the PHP treatment. Evidences suggested that phenolic acid, FOs and curcumin could upregulated the phase II detoxifying/antioxidant enzymes expression by activation of the Nrf2/ARE pathway both in vitro and in vivo. ${ }^{6,35,40}$ In one word, the modulation of CAT, SOD, GSH-Px and Nrf2/ARE signaling of PHP were testified in this study. PHP could cause a dissociation of Nrf2-Keap1 complex, induce the Nrf2 phosphorylation, and lead to formation of the Nrf2-MafK heterodimers, which promote transcriptional activation of phase II antioxidant/detoxifying enzymes by binding to ARE (Fig. 5).

\section{Conclusions}

This study demonstrated that PHP identified from Chinese Baijiu possessed preventive effects against AAPH-induced oxidative stress in HepG2 cells by suppressing ROS generation, preventing MDA formation, and up-regulating cellular antioxidant enzyme activity, including SOD, CAT, and GSH-Px. When the concentration range was $2.00 \mathrm{mg} \mathrm{mL} \mathrm{m}^{-1}$, PHP exhibited more powerful antioxidant activity than Trolox. In order to explore the possible antioxidant mechanism of PHP, the mRNA and protein expression treated by different levels of PHP were determined. The results showed PHP treatment stimulated the activities, mRNA expression levels, and protein expression levels of antioxidant enzymes and Nrf2, which suggested that PHP effectively activated Nrf2/ARE-mediated activity. These findings provide the first molecular basis for the health-promoting effects of PHP to prevent AAPH-induced oxidative stress, and may be useful in functional Baijiu 
industry. It was also important for us to understand further the bioactive peptides in Baijiu.

\section{Conflicts of interest}

The authors declare no conflict of interest.

\section{Acknowledgements}

This work was supported by the National Key Research \& Development Program of China (2016YFD0400500); and the National Natural Science Foundation of China (31471665 and 31701567).

\section{References}

1 K. Itoh, K. I. Tong and M. Yamamoto, Free Radicals Biol. Med., 2004, 36(10), 1208-1213.

2 Y. J. Surh, J. K. Kundu and H. K. Na, Planta Med., 2008, 74, 1526-1539.

3 J. K. Kundu and Y. J. Surh, Pharm. Res., 2010, 27, 999-1013. 4 T. H. Rushmore, R. G. King, K. E. Paulson and C. B. Pickett, Proc. Natl. Acad. Sci. U. S. A., 1990, 87(10), 3826-3830.

5 T. Nguyen, P. Nioi and C. B. Pickett, J. Biol. Chem., 2009, 284(20), 13291-13295.

6 H. Zhang, S. Zhang, J. Wang and B. Sun, J. Funct. Foods, 2017, 29, 53-59.

7 M. Á. Martín, A. B. G. Serrano, S. Ramos, M. I. Pulido, L. Bravo and L. Goya, J. Nutr. Biochem., 2010, 21(3), 196-205.

8 E. O. Farombi, S. Shrotriya, H. Na, S. Kim and Y. Surh, Food Chem. Toxicol., 2008, 46, 1279-1287.

9 J. K. Kim and H. D. Jang, Int. J. Mol. Sci., 2014, 15(7), 1214912165.

10 M. La Marca, P. Beffy, C. Della Croce, P. G. Gervasi, R. Iori, E. Puccinelli and V. Longo, Food Chem. Toxicol., 2012, 50(8), 2822-2830.

11 H. M. Chen, K. Muramoto and F. Yamauchi, J. Agric. Food Chem., 1995, 43, 574-578.

12 R. R. Lu, P. Qian, Z. Sun, X. H. Zhou, T. P. Chen, J. F. He, H. Zhang and J. P. Wu, Food Chem., 2010, 123, 1210-1218.

13 W. Y. Huang, K. Majumder and J. Wu, Food Chem., 2010, 123, 635-641.

14 J. Wu, B. Sun, M. Zhao, F. Zheng, J. Sun, X. Sun, H. Li and M. Huang, J. Chin. Inst. Food Sci. Technol., 2016, 16(9), 14-20.

15 H. Zhang, J. Wang, Y. Liu, L. Gong and B. Sun, J. Funct. Foods, 2016, 25, 333-340.

16 T. Fukuda, K. Yamagata, S. Fujiyama, T. Matsumoto, I. Koshida, K. Yoshimura and S. Kato, Nat. Cell Biol., 2007, 9(5), 604-611.
17 W. Yu, G. Chen, P. Zhang and K. Chen, Int. J. Biol. Macromol., 2016, 82, 299-307.

18 G. Wang, Z. Lei, Q. Zhong, W. Wu, H. Zhang, T. Min, H. Wu and A. F. Lai, Food Chem., 2017, 217, 332-341.

19 J. Wu, J. Huo, M. Huang, M. Zhao, X. Luo and B. Sun, J. Agric. Food Chem., 2017, 65, 10495-10504.

20 K. J. Livak and T. D. Schmittgen, Methods: A Companion to Methods in Enzymology, 2001, 25(4), 402-408.

21 C. T. Yeh and G. C. Yen, J. Nutr., 2006, 136(1), 11-15.

22 D. Zhao, J. Sun, B. Sun, M. Zhao, F. Zheng, M. Huang, X. Sun and H. Li, RSC Adv., 2017, 7, 46395-46405.

23 L. Wang, L. Ding, Z. Yu, T. Zhang, S. Ma and J. Liu, Food Res. Int., 2016, 90, 33-41.

24 H. Agrawal, R. Joshi and M. Gupta, Food Chem., 2016, 204, 365-372.

25 Y. Du, R. Esfandi, W. Willmore and A. Tsopmo, Antioxidants, 2016, 5(39), 1-9.

26 H. Liu, J. Ma and H. Wu, RSC Adv., 2017, 7, 48913-48924.

27 M. Zhang, Z. Hong, H. Li, F. Lai, X. F. Li, Y. Tang, M. Tian and W. Hui, J. Agric. Food Chem., 2016, 64, 7921-7930.

28 W. Liao, Z. Ning, L. Chen, Q. Wei, E. Yuan, J. Yang and J. Ren, J. Agric. Food Chem., 2014, 62, 8648-8654.

29 S. Katayama, S. Ishikawa, M. Fan and Y. Mine, J. Agric. Food Chem., 2007, 55, 2829-2835.

30 Y. Kim, Y. Choi, H. Ham, H. Jeong and J. Lee, Food Chem., 2013, 137, 136-141.

31 J. Wang, B. Sun, Y. Cao and C. Wang, Food Chem., 2010, 123, 472-476.

32 M. Alía, S. Ramos, R. Mateos, A. Granado-Serrano, L. Bravo and L. Goya, Toxicol. Appl. Pharmacol., 2006, 212, 110-118.

33 M. Lee, B. Lee, K. Park, T. Utsuki, T. Shin, C. W. Oh and H. Kim, Food Chem., 2015, 174, 538-546.

34 G. Qi, Y. Mi, R. Fan, R. Li, Y. Wang, X. Li, S. Huang and A. X. Liu, $R S C A d v ., 2017,7,32198-32208$.

35 G. N. Kim, Y. J. Lee, J. H. Song and H. D. Jang, Food Sci. Biotechnol., 2013, 22(1), 241-247.

36 S. Tanigawa, M. Fujii and D. Hou, Free Radical Biol. Med., 2007, 42, 1690-1703.

37 Z. Zhang, S. Zhou, X. Jiang, Y. Wang, F. Li, Y. Wang, Y. Zheng and L. Cai, Rev. Endocr. Metab. Disord., 2015, 16, 35-45.

38 E. Balogun, M. Hoque, P. Gong, E. Killeen, C. J. Green, R. Foresti, J. Alam and R. Motterlini, Biochem. J., 2003, 371(3), 887-895.

39 S. Ye, Z. Hou, L. Zhong and Q. Zhang, Yao Xue Xue Bao (Acta Pharmaceutica Sinica), 2007, 42(4), 376-380.

40 F. Shahidi, P. Janitha and P. Wanasundara, Phenolic antioxidants, Crit. Rev. Food Sci. Nutr., 1992, 32(1), 67-103. 